\title{
Ein kurzes Nachwort
}

Am Ende der beiden Bücher ist nun der Moment gekommen, sich die Frage zu stellen, ob sich die ganze Aktion gelohnt hat. In Summe waren es acht Jahre Arbeit mit sehr hoher Intensität, ganz besonders zum Schluss. Es scheint aber Studentinnen und Studenten zu geben, denen diese Bücher geholfen haben, und daher bin ich mit diesem Projekt zufrieden. Ganz besonders zufrieden bin ich damit, dass es auch einen unglaublichen Feedback von studentischer Seite gab, und noch immer gibt, den ich niemals erwartet hätte. Mein aufrichtiger Dank ergeht nochmals an alle, die mitgemacht haben, und an die, die auch heute noch mitmachen.

Teil II des Buches hat Ihnen hoffentlich einen effizienten Einstieg in das Gebiet der niedrig-dimensionalen Elektronensysteme und deren Anwendungen ermöglicht. Aber, wie ganz am Anfang erwähnt, wir haben uns wieder nur an der Oberfläche der Thematik bewegt. Wenn Sie mehr wissen wollen, als in diesem Buch steht, werden Sie wohl nun doch die restlichen paar tausend Seiten zu diesem Thema im Internet selber studieren müssen.

Für mich gilt weiterhin das Motto: Dieses Buch soll, wenn es geht, besser werden, aber nicht substantiell länger. Jeder Feedback (via E-mail an juergen.smoliner@ tuwien.ac.at) ist willkommen, und jeder Beitrag kommt namentlich auf die Liste der Helden von Haegrula im Dank. Ein aktuelles Vorab-Exemplar der nächsten Auflage des Buches bekommen Sie selbstverständlich und gratis per E-mail von mir. Da hat auch der Springer Verlag nichts dagegen.

Viel Erfolg beim Studium und beste Grüße aus Wien, Jürgen Smoliner 